MATHEMATICS OF COMPUTATION

Volume 74, Number 251, Pages 1391-1411

S 0025-5718(04)01694-1

Article electronically published on July 20, 2004

\title{
INSTABILITY ANALYSIS OF SADDLE POINTS BY A LOCAL MINIMAX METHOD
}

\author{
JIANXIN ZHOU
}

\begin{abstract}
The objective of this work is to develop some tools for local instability analysis of multiple critical points, which can be computationally carried out. The Morse index can be used to measure local instability of a nondegenerate saddle point. However, it is very expensive to compute numerically and is ineffective for degenerate critical points. A local (weak) linking index can also be defined to measure local instability of a (degenerate) saddle point. But it is still too difficult to compute. In this paper, a local instability index, called a local minimax index, is defined by using a local minimax method. This new instability index is known beforehand and can help in finding a saddle point numerically. Relations between the local minimax index and other local instability indices are established. Those relations also provide ways to numerically compute the Morse, local linking indices. In particular, the local minimax index can be used to define a local instability index of a saddle point relative to a reference (trivial) critical point even in a Banach space while others failed to do so.
\end{abstract}

\section{INTRODUCTION}

Multiple solutions with different performance, maneuverability and instability indices exist in many nonlinear problems in the natural or social sciences [1, 7, 9. 24, 29, 31, 33, 35. When cases are variational, the problems reduce to solving the Euler-Lagrange equation

$$
J^{\prime}(u)=0
$$

where $J$ is a $C^{1}$ generic energy functional on a Banach space $H$ and $J^{\prime}$ is its Frechet derivative. A solution $u^{*} \in H$ to the Euler-Lagrange equation (1.1) is called a critical point of $J$. Its value $J\left(u^{*}\right)$ is called a critical value. A critical point $u^{*}$ of $J$ is nondegenerate if the second Frechet derivative $J^{\prime \prime}\left(u^{*}\right)$ exists and is invertible as a linear operator. Otherwise $u^{*}$ is said to be degenerate. The first candidates for a critical point are naturally the local extrema to which the classical critical point theory was devoted in calculus of variation. Most conventional numerical algorithms focus on finding such stable solutions. Critical points that are not local extrema are unstable and are called saddle points, that is, critical points $u^{*}$ of $J$ s.t. any neighborhood of $u^{*}$ in $H$ contains points $v, w$ satisfying $J(v)<J\left(u^{*}\right)<J(w)$. In physical systems, saddle points appear as unstable equilibria or transient excited states.

Received by the editor May 4, 2003 and, in revised form, December 11, 2003.

2000 Mathematics Subject Classification. Primary 58E05, 58E30; Secondary 35A40, 35A15.

Key words and phrases. Saddle point, instability index, Morse index, (weak) local linking, local minimax method.

(C)2004 American Mathematical Society 
When multiple solutions exist in a nonlinear system, some are stable and others are unstable. Among those unstable solutions, their instability behavior can be very different. Stability/instability is one of the main concerns in system design and control theory. On the other hand, in many applications, performance or maneuverability is more desirable. An unstable solution can be stable enough to accomplish a short term mission before being excited to decay to another desirable state. Unstable solutions may have much higher maneuverability or performance indices, which could be crucial in a mission critical situation. For instance, traveling waves have been observed to exist in suspended bridges [9] and have been shown to be saddle points, therefore unstable solutions, to their corresponding variational problem ((10) and (11) in 9]). Those unstable solutions have been observed to have quite different amplitudes and instability behavior. Vector solitons arise in many fields, such as condensed matter physics, dynamics of biomolecules, nonlinear optics, etc. For example, in the study of self-guided light waves in nonlinear optics [14, 15, 25], excited states are of great interests. All those solitons are saddle points, thus unstable solutions. Among them, solutions which are not ground states, are the so-called excited states. Among many different modes of excited states are the vortex-mode and dipole-mode vector solitons. It has been experimentally and numerically proved that these two unstable solutions have very different instability and maneuverability properties. The vortex-mode can be easily perturbed to decay into a dipole-mode. While the dipole-modes are much more stable, "stable enough for experimental observation, ..., extremely robust, have a typical lifetime of several hundred diffraction lengths and survive a wide range of perturbations" [14, they thus are hard to excite.

Can one find those multiple solutions and measure their instabilities or maneuverabilities? This is certainly an interesting engineering problem in system design and control.

The objective of this work is to develop some tools for local instability analysis of saddle points which can be computationally carried out. To fulfill the objective, first one has to find a method to numerically approximate an unstable solution in a stable way. This is a vary challenging task. Previous works [20, 21] have laid a solid foundation. Then one needs to find a way to measure local instabilities of saddle points. Usually this is done by defining a certain local instability index. Here we want to define a local instability index for a saddle point which is general enough to be applied to usual cases and meanwhile can be easily numerically computed.

There is a well-known theorem in ordinary differential equations, tracing back to Poincaré, which states that the stability of a rest point can be inferred from "linearization." More precisely, if one considers the ordinary differential equation $u^{\prime}=f(u)$ in $\mathbb{R}^{n}$ with $\bar{u}$ a rest point, i.e., $f(\bar{u})=0$, the linearization equation is $u^{\prime}=d f(\bar{u}) u$ and $u=0$ is a rest point. Then if the differential $d f(\bar{u})$ (an $n \times n$ matrix) has all of its eigenvalues in the left-hand plane, $\operatorname{Re} z<0$, it follows that $\bar{u}$ is asymptotically stable; i.e., if $u_{0}$ is near $\bar{u}$, then the solution of the equation through $u_{0}$ tends to $\bar{u}$ as $t \rightarrow+\infty$. This idea has been extended to the study of (in)stability of solutions to variational problems (Euler-Lagrange equation), such as a stationary (time-harmonic) solution to a semilinear reaction-diffusion or hyperbolic equation, standing wave or traveling wave solutions to many nonlinear wave phenomena, e.g., the nonlinear Schrodinger equation $i v_{t}+\Delta v+f(|v|) v=0$, or, periodic solutions 
to many nonlinear Hamiltonian systems and nonlinear wave equation, e.g.,

$$
\begin{cases}u_{t t}=u_{x x}-f(u), & 0<x<\pi, 0<t<T, \\ u(0, t)=u(\pi, t)=0, & u(x, t+T)=u(x, t),\end{cases}
$$

to which a solution $u^{*}$ corresponds to a critical point of the generic energy functional

$$
J(u)=\int_{0}^{T} \int_{0}^{\pi}\left(\frac{1}{2}\left(\left|u_{x}\right|^{2}-\left|u_{t}\right|^{2}\right)-F(u)\right) d x d t,
$$

where $F(r)=\int_{0}^{r} f(\tau) d \tau$.

Let $u^{*}$ be a critical point of $J$. Consider the negative gradient flow of $J$ at $u^{*}$ defined by

$$
\left\{\begin{aligned}
\frac{d}{d \tau} \sigma(\tau, u) & =-\nabla J(\sigma(\tau, u)), \\
\sigma(0, u) & =u^{*} .
\end{aligned}\right.
$$

Then it is clear that $\sigma(\tau, u) \equiv u^{*}$ is a rest point of (1.3). When $J^{\prime \prime}\left(u^{*}\right)$ is nonsingular, $u^{*}$ is a nondegenerate rest point. Now we consider the flow for the "linearization equation"

$$
v^{\prime}=-J^{\prime \prime}\left(u^{*}\right) v .
$$

Clearly, $v=0$ is a rest point of (1.4). When $J^{\prime \prime}\left(u^{*}\right)$ is self-adjoint, its eigenvalues are all real. Thus if all eigenvalues of $J^{\prime \prime}\left(u^{*}\right)$ are positive, then $u^{*}$ is a stable solution. Otherwise $u^{*}$ is unstable and the number of negative eigenvalues of $J^{\prime \prime}\left(u^{*}\right)$, i.e., the Morse index of $u^{*}$, can be used as an index to measure the "instability" [31. However, when $J^{\prime \prime}\left(u^{*}\right)$ is singular, the above argument fails. Note that starting from a critical point $u^{*}$, along an eigenvector corresponding to a negative (positive) eigenvalue of $J^{\prime \prime}\left(u^{*}\right), J$ is decreasing (increasing).

Definition 1.1. A vector $v \in H$ is said to be a decreasing (increasing) direction of $J$ at a critical point $u^{*} \in H$ if there exists $T>0$ s.t.

$$
J\left(u^{*}+t v\right)<(>) J\left(u^{*}\right) \quad \forall T>t>0 .
$$

In general, the set of all decreasing (or increasing) vectors of $J$ at a critical point does not form a linear vector space. The maximum dimension of a subspace of decreasing directions of $J$ at a critical point $u^{*}$ is called the local instability index of $J$ at $u^{*}$.

Since such an index lacks characterization and is too difficult to compute, let us study several alternatives.

Morse index. According to spectral theory, when $J^{\prime \prime}\left(u^{*}\right)$ exists and is a selfadjoint Fredholm operator from $H \rightarrow H$ at a critical point $u^{*}$, the Hilbert space $H$ has an orthogonal spectral decomposition

$$
H=H^{-} \oplus H^{0} \oplus H^{+}
$$

where $H^{-}, H^{0}$ and $H^{+}$are, respectively, the maximum negative definite subspace, the null subspace and the maximum positive definite subspace of $J^{\prime \prime}\left(u^{*}\right)$ in $H$ with $\operatorname{dim}\left(H^{0}\right)<\infty$ and are all closed invariant subspaces under $J^{\prime \prime}\left(u^{*}\right)$. Following Morse theory, the Morse index of the critical point $u^{*}$ is $\operatorname{MI}\left(u^{*}\right)=\operatorname{dim}\left(H^{-}\right)$. It is clear that a nondegenerate critical point $u^{*}$ with $\mathrm{MI}\left(u^{*}\right)=0$ is a local minimum point of the generic energy function $J$ and therefore is a stable solution, and a critical point $u^{*}$ of $J$ with $\operatorname{MI}\left(u^{*}\right)>0$ is an unstable solution. When $u^{*}$ is nondegenerate, i.e., $H^{0}=\{0\}, \operatorname{MI}\left(u^{*}\right)$ is the local instability index of $J$ at $u^{*}$. Thus the Morse 
index has been used [31] to measure local instabilities of basically nondegenerate critical points. Numerical computation of the Morse index of a saddle point $u^{*}$ basically consists of two steps, first to numerically compute the unstable solution $u^{*}$ and then to numerically solve for the number of negative eigenvalues of the corresponding linearized problem at $u^{*}$. It is very expensive. Therefore researchers (see, e.g., [2], [3], [5], 7], 17], 30], [32, and others) tried to establish some (bound) estimates of the Morse index of saddle points based on certain (global) minimax characterizations. On the other hand, the Morse index is ineffective in measuring local instability of a degenerate critical point, since many different situations may happen in the nullspace $H^{0}$.

Local linking index. A local linking defined in [19 can also be used to define a local instability index (LLI) for saddle points that are not necessarily nondegenerate.

Definition 1.2 (19]). A function $J \in C^{1}(H, R)$ has a local linking at $u^{*} \in H$ if there exist subspaces $H^{I}, H^{D}$ in $H$ and a constant $r>0$ s.t. $H=H^{I} \oplus H^{D}$ and

$$
\begin{aligned}
& J\left(u^{*}+u_{1}\right) \geq J\left(u^{*}\right), \forall u_{1} \in H^{I}, \text { with } 0<\left\|u_{1}\right\| \leq r, \\
& J\left(u^{*}+u_{2}\right) \leq J\left(u^{*}\right), \forall u_{2} \in H^{D}, \text { with } 0<\left\|u_{2}\right\| \leq r .
\end{aligned}
$$

It is clear that each point $u \neq 0$ in $H^{I}\left(H^{D}\right)$ is an increasing (decreasing) direction of $J$ at $u^{*}$ and $H^{I}\left(H^{D}\right)$ is the space of increasing (decreasing) directions of $J$ at $u^{*}$. We may consider using $\operatorname{dim}\left(H^{D}\right)$ as a local instability index (LLI). The notion of a local linking is introduced by $\mathrm{Li}$ and Liu in 18 and then modified by Li and Willem in [19] to prove the existence of multiple critical points. It is extensively used with $\operatorname{dim}\left(H^{D}\right)<\infty$ in 30 by Silva to establish the existence of multiple critical points and to estimate their Morse indices. We can see that if $J$ has a local linking at $u^{*}$, then $u^{*}$ is a critical point of $J$.

According to the Morse lemma 7, the uniform bound $r$ in the definition of a local linking can be obtained if $J$ is $C^{2}$ and $u^{*}$ is a nondegenerate critical point. Thus a nondegenerate saddle point is a local linking with $H^{-}=H^{D}$ and $H^{+}=H^{I}$. For a degenerate saddle point if we have $H^{0}=H^{0 D} \oplus H^{0 I}$ where $H^{0 D}$ and $H^{0 I}$ consist, respectively, of decreasing and increasing directions of $J$ at $u^{*}$, then $u^{*}$ is also a local linking with $H^{D}=H^{-} \oplus H^{O D}, H^{I}=H^{+} \oplus H^{0 I}$. However, in general such a splitting is not available.

\section{Example 1.1. Let}

$$
J_{1}\left(x_{1}, x_{2}\right)=\left(x_{1}-x_{1}^{*}\right)^{2}-\left(x_{2}-x_{2}^{*}\right)^{2} \text { and } J_{2}\left(x_{1}, x_{2}\right)=\left(x_{1}-x_{1}^{*}\right)^{2}-\left(x_{2}-x_{2}^{*}\right)^{4} \text {. }
$$

Then $x^{*}=\left(x_{1}^{*}, x_{2}^{*}\right)$ is a critical point for both $J_{1}$ and $J_{2}$. However, for $J_{1}$, MI $\left(x^{*}\right)=$ $\operatorname{LLI}\left(x^{*}\right)=1$ and for $J_{2}, 0=\operatorname{MI}\left(x^{*}\right)<\operatorname{LLI}\left(x^{*}\right)=1$. Due to degeneracy, the Morse index fails to describe the local instability of $J_{2}$ at $x^{*}$. While LLI serves well.

Example 1.2. For $H=\mathbb{R}^{3}$, let $J\left(x_{1}, x_{2}, x_{3}\right)=x_{1}^{2}-x_{3}^{2}$. Then 0 is a critical point with $H^{+}=\left\{e_{1}\right\}, H^{0}=\left\{e_{2}\right\}$ and $H^{-}=\left\{e_{3}\right\}$. This is a degenerate case, since $J$ is constant along the direction $e_{2}$. In this case, we may either set $H^{I}=\left\{e_{1}, e_{2}\right\}, H^{D}=$ $\left\{e_{3}\right\}$ or set $H^{I}=\left\{e_{1}\right\}, H^{D}=\left\{e_{2}, e_{3}\right\}$. In either case, $H^{I}$ is an increasing space and $H^{D}$ is a decreasing space and 0 is a local linking with $H=H^{I} \oplus H^{D}$. But two $H^{D}$ 's have a dimension difference 1 . Thus the LLI index at 0 is not well defined. If we limit the inequality in (1.8) to be strict, then the second case will be eliminated. There will be no ambiguity. 
The definition of a local linking provides a generalization of the Morse index to a degenerate critical point. However, the dimensions of the spaces $H^{I}$ and $H^{D}$ in the definition are not uniquely determined. We also note that the uniform bound $r$ in the definition of a local linking is difficult to obtain when either $H^{I}$ or $H^{D}$ is infinite-dimensional, in particular, at a degenerate saddle point. Therefore it is reasonable for us to weaken the uniform bound condition and to allow individual bounds. We introduce the following definition.

Definition 1.3. A function $J \in C^{1}(H, R)$ has a weak local linking at $u^{*} \in H$ if $H=H^{I} \oplus H^{D}$ for some subspaces $H^{I}, H^{D}$ in $H$ and for each $u_{1} \in H^{I}$ and $u_{2} \in H^{D}$ with $\left\|u_{1}\right\|=1$ and $\left\|u_{2}\right\|=1$ there exist constants $r_{1}>0$ and $r_{2}>0$ s.t.

$$
\begin{aligned}
& J\left(u^{*}+t u_{1}\right) \geq J\left(u^{*}\right), \forall 0<|t| \leq r_{1}, \\
& J\left(u^{*}+t u_{2}\right)<J\left(u^{*}\right), \forall 0<|t| \leq r_{2} .
\end{aligned}
$$

We define the local linking index (LLI) of $J$ at $u^{*}$ by

$$
\operatorname{LLI}\left(u^{*}\right) \equiv \operatorname{dim}\left(H^{D}\right) .
$$

Lemma 1.1. If $J$ has a weak local linking at $u^{*}$, then $L L I\left(u^{*}\right)$ is well defined.

Proof. We only have to show that if $H=\tilde{H}^{I} \oplus \tilde{H}^{D}$ for some subspaces $\tilde{H}^{I}, \tilde{H}^{D}$ in $H$ and for each $u_{1} \in \tilde{H}^{I}$ and $u_{2} \in \tilde{H}^{D}$ with $\left\|u_{1}\right\|=1$ and $\left\|u_{2}\right\|=1$ there exist constants $r_{1}>0$ and $r_{2}>0$ s.t.

$$
\begin{aligned}
& J\left(u^{*}+t u_{1}\right) \geq J\left(u^{*}\right), \forall 0<|t| \leq r_{1}, \\
& J\left(u^{*}+t u_{2}\right)<J\left(u^{*}\right), \forall 0<|t| \leq r_{2},
\end{aligned}
$$

then $\operatorname{dim}\left(\tilde{H}^{D}\right)=\operatorname{dim}\left(H^{D}\right)$. Suppose $\operatorname{dim}\left(\tilde{H}^{D}\right)<\operatorname{dim}\left(H^{D}\right)$. We have $\tilde{H}^{I} \cap H^{D} \neq$ $\{0\}$. But this is impossible, since $\tilde{H}^{I}$ is an increasing direction space of $J$ at $u^{*}$ and $H^{D}$ is a strictly decreasing direction space of $J$ at $u^{*}$.

The definition of a local linking lacks characterization and it is still too difficult to compute numerically. So far no constructive method to compute such an index is available in the literature.

In this paper, we use a local minimax method developed in 20, 21] to define a new local instability index which is known beforehand and can help in finding a saddle point numerically.

Throughout this paper, when the Morse index is involved, we always assume that $J^{\prime \prime}\left(u^{*}\right)$ is a self-adjoint Fredholm linear operator from $H \rightarrow H$ where $u^{*}$ is a critical point of $J$. Thus the orthogonal spectral decomposition (1.6) is always available; when a local linking is involved, we always assume that $H=H^{I} \oplus H^{D}$ where $H^{I}$ and $H^{D}$ are, respectively, the increasing direction subspace and the maximum decreasing direction subspace as stated in (1.9) and (1.10).

\section{A LOCAL MinimaX INDEX AND its RELATion to Mi AND LLI}

Since Ljusternik-Schnirelmann, under a deformation assumption, proved (1930) the existence of a saddle point as a minimax solution, i.e., a solution to

$$
\min _{A \in \mathcal{A}} \max _{v \in A} J(v)
$$

where $\mathcal{A}$ is a collection of (compact) subsets in $H$, the minimax principle becomes one of the most popular approaches in critical point theory. 
The Mountain Pass Lemma proved (1973) by Ambrosetti and Rabinowitz [1] set a milestone in nonlinear analysis. Since then, the subject area has undergone explosive growth. Many minimax theorems, such as various linking and saddle point theorems, have been successfully established to prove the existence of multiple solutions to various nonlinear PDE's and dynamic systems [1, 6], [4], 10], [11, [12], [16], 23, 24], 27], [28, [29], 33], [34], [35. But almost all minimax theorems in critical point theory focus only on the existence issue. They require one to solve a two-level global optimization problem and therefore are not for algorithm implementation and they cannot precisely describe the local instability behavior of a saddle point.

Inspired by the numerical works of Choi and McKenna [8] and Ding, Costa, and Chen [13], and motivated by Morse theory and the idea of defining a (stable) solution manifold [26], [27, [28, a local minimax method is developed in [20] which characterizes a saddle point as a local minimax solution. Based on the local minimax characterization, a numerical local minimax algorithm is developed and successfully applied to solve many semilinear elliptic PDE on various domains for multiple solutions [20], 21]. In this paper, we show that the local minimax method can be used to define an index to measure the local instability of a local minimax solution in a way much better than the Morse and other (local linking) indices. Furthermore this index is known even before we numerically compute the local minimax solution.

Let $H$ be a Hilbert space. For a subspace $H^{\prime} \subset H$, denote $S_{H^{\prime}}=\{v \mid v \in$ $\left.H^{\prime},\|v\|=1\right\}$. Let $L$ be a closed subspace, called a support, in $\mathrm{H}$ and let $L^{\perp}$ be its orthogonal complement in $H$. Thus $H=L \bigoplus L^{\perp}$. For each $v \in S_{L^{\perp}}$, denote $\{L, v\}=\left\{t v+v_{L} \mid t \in \mathbb{R}, v_{L} \in L\right\}$.

Definition 2.1. A set-valued mapping $P: S_{L^{\perp}} \rightarrow 2^{H}$ is called the peak mapping of $J$ w.r.t. $H=L \bigoplus L^{\perp}$ if for any $v \in S_{L^{\perp}}, P(v)$ is the set of all local maximum points of $J$ in $\{L, v\}$. A single-valued mapping $p: S_{L^{\perp}} \rightarrow H$ is a peak selection of $J$ w.r.t. $L$ if $p(v) \in P(v) \quad \forall v \in S_{L^{\perp}}$. Let $v \in S_{L^{\perp}}$. Then $J$ is said to have a local peak selection $p$ w.r.t. $L$ at $v$ if there exist a neighborhood $\mathcal{N}(v)$ of $v$ and a mapping $p: \mathcal{N}(v) \cap S_{L^{\perp}} \rightarrow H$ s.t.

$$
p(u) \in P(u) \quad \forall u \in \mathcal{N}(v) \cap S_{L^{\perp}} .
$$

In [20], [21], the peak mapping $P(v)$ for $v \in S_{L^{\perp}}$ is defined by using the half space $[L, v]=\left\{t v+v_{L}: t \geq 0, v_{L} \in L\right\}$ and not the whole space $\{L, v\}$ due to the fact that the model problem considered there has a certain symmetric property. When more general cases are considered, it is better to use the whole space. The corresponding change is minor; however, it makes the proof of nonemptiness of $P(v)$ easier.

Theorem 2.1 ([20]). Let $v^{*} \in S_{L^{\perp}}$ be a point. If $J$ has a local peak selection $p$ w.r.t. $L$ at $v^{*}$ s.t. (i) $p$ is continuous at $v^{*}$, (ii) $p\left(v^{*}\right) \notin L$ and (iii) $v^{*}$ is a local minimum point of $J(p(v))$ on $S_{L^{\perp}}, u^{*}=p\left(v^{*}\right)$ is a critical point of $J$.

Note that in the above theorem both the maximization, i.e., $p\left(v^{*}\right)$, at the first level and the minimization at the second level are locally defined. If we define a (stable) solution set

$$
\mathcal{M}=\left\{p(v): v \in S_{L^{\perp}}\right\}
$$

then a local minimum point of $J$ on $\mathcal{M}$ is a critical point and can be approximated, e.g., by a steepest descent search [20] and [21]. 
Now we have three types of decreasing direction spaces of $J$ at $u^{*}$, namely, $\left\{L, v^{*}\right\}, H^{-}$and $H^{D}$. The dimensions of those three spaces can be used to measure local instability at $u^{*}$. Their relations will be explored.

In this paper, we use the local minimax characterization to classify those saddle points with which a local instability index can be assigned. In particular, we show that once a local minimax solution is found by the local minimax method, a local instability index of the solution is automatically known

Definition 2.2. A critical point $u^{*}$ of $J$ is said to be a (local) minimax solution, if there is a closed subspace (support) $L \subset H$, a point $v^{*} \in S_{L^{\perp}}$ and a local peak selection $p$ of $J$ w.r.t. $L$ at $v^{*}$ s.t. $u^{*}=p\left(v^{*}\right)$ and

$$
v^{*}=\arg \min _{v \in S_{L^{\perp}}} J(p(v))=\arg \min _{v \in S_{L^{\perp}}} \max _{u \in\{L, v\}} J(u),
$$

where the local peak selection $p$ is continuous at $v^{*}$ and either the local maximum at the first level or the local minimum at the second level is strict.

Lemma 2.1. If $u^{*}$ is a minimax solution of $J$ w.r.t. the support L s.t. $u^{*} \notin L$, then $L$ is maximum in the sense that any proper subspace $L^{\prime}$ of $L$ is not a support with which $u^{*}$ is a minimax solution.

Proof. Let $u^{*}=p\left(v^{*}\right)$ be a minimax solution w.r.t. the support $L$ where $v^{*} \in S_{L^{\perp}}$ and $p$ is a local peak selection continuous at $v^{*}$ and meanwhile let $u^{*}=\tilde{p}(\tilde{v})$ also be a minimax solution w.r.t. the support $\tilde{L}$ where $\tilde{v} \in S_{\tilde{L}^{\perp}}$ and $\tilde{p}$ is a local peak selection continuous at $\tilde{v}$. Suppose $\tilde{L} \subset L$ is proper. We have $\tilde{L}^{\perp} \cap L \neq\{0\}$. Choose any $v \in \tilde{L}^{\perp} \cap L$ with $\|v\|=1$. Since $\tilde{v} \in \tilde{L}^{\perp}$, for any real number $s$ with $|s|$ small, set

$$
\tilde{v}(s)=\frac{\tilde{v}+s v}{\|\tilde{v}+s v\|} \in \mathcal{N}(\tilde{v}) \cap S_{\tilde{L}^{\perp}},
$$

where $\mathcal{N}(\tilde{v})$ is a neighborhood of $\tilde{v}$ in which the local peak selection $\tilde{p}$ is defined. We have

$$
J(\tilde{p}(\tilde{v}(s)))>(\geq) J(\tilde{p}(\tilde{v}))=J\left(u^{*}\right) .
$$

On the other hand, since $u^{*}=p\left(v^{*}\right) \notin L$, we have

$$
u^{*} \equiv p\left(v^{*}\right)=t^{*} v^{*}+v_{L}^{*} \quad \text { and } \quad u^{*} \equiv \tilde{p}(\tilde{v})=\tilde{t} \tilde{v}+\tilde{v}_{\tilde{L}}
$$

for some nonzero scalars $t^{*}$ and $\tilde{t}$, and points $v_{L}^{*} \in L$, and $\tilde{v}_{\tilde{L}} \in \tilde{L} \subset L$. Equating the last two expressions, we obtain $\tilde{v} \in\left\{L, v^{*}\right\}$. Note that $\tilde{p}(\tilde{v}(s))=\tilde{t}_{s} \tilde{v}(s)+\tilde{v}_{\tilde{L}}(s)$ for some scalar $\tilde{t}_{s}$ and point $\tilde{v}_{\tilde{L}}(s) \in \tilde{L} \subset L$, and $\tilde{v} \in\left\{L, v^{*}\right\}$ and $v \in \tilde{L}^{\perp} \cap L$ imply

$$
\tilde{p}(\tilde{v}(s)) \in\left\{\left\{L, v^{*}\right\}, v\right\} \subset\left\{L, v^{*}\right\} .
$$

Since $\tilde{v}(s) \rightarrow \tilde{v}$ as $s \rightarrow 0$ and $\tilde{p}$ is continuous at $\tilde{v}$, we have

$$
\tilde{p}(\tilde{v}(s)) \rightarrow \tilde{p}(\tilde{v})=u^{*}=p\left(v^{*}\right) \quad \text { as } \quad s \rightarrow 0 .
$$

Now $u^{*}=p\left(v^{*}\right)$ is a local maximum point of $J$ in $\left\{L, v^{*}\right\}$; when $|s|>0$ is small, we have

$$
J(\tilde{p}(\tilde{v}(s))) \leq(<) J\left(p\left(v^{*}\right)\right)=J\left(u^{*}\right),
$$

which contradicts (2.1). Thus $L=\tilde{L}$, i.e., $L$ is maximum.

Definition 2.3. Let $u^{*}$ be a local minimax solution of $J$ w.r.t. the support $L$. The local minimax index (MMI) of the saddle point $u^{*}$ w.r.t. $L$ is defined by

$$
\text { MMI }\left(u^{*}\right) \equiv \operatorname{dim}\left\{L, v^{*}\right\} \equiv \operatorname{dim}(L)+1 \text {. }
$$


Note that in general a support for a local minimax solution is not unique. We may define a local minimax index of $u^{*}$ by assuming that $L$ is the one with either maximum or minimum dimension among all the supports. However, in this paper, we focus on what information the integer $\operatorname{dim}(L)+1$ can tell about local instability of $u^{*}$. When $L$ is spanned by previously found critical points at a lower critical level, $u^{*}$ can be perturbed by vectors in $\left\{L, v^{*}\right\}$ to decay into a critical point at a lower critical level. Thus, the number $\operatorname{dim}(L)+1$ can also be used to measure the maneuverability of $u^{*}$.

Following the local minimax method, MMI $\left(u^{*}\right)$ is known even before we numerically find the solution $u^{*}$. Note that MMI may not be defined for a general critical point. However, we will show that for a class of familiar critical points, MMI is well defined. Usually when an index is defined to a solution, to compute the index, one has to find the solution first and then follow certain rules or a certain method to compute the index of the solution, e.g., the Morse and local linking indices. Experience tells us, this is usually difficult and expensive. Here we reverse the process. The local minimax method utilizes the geometric and topological structure of the local minimax index to numerically compute a critical point with such an index.

We now start to establish some relations between MMI, MI and LLI.

Theorem 2.2. If $u^{*}=t^{*} v^{*}+v_{L}^{*}$ for some $v^{*} \in S_{L^{\perp}}, t^{*} \neq 0$ and $v_{L}^{*} \in L$ is a critical point of $J$ s.t. $u^{*}$ is not a local minimum point of $J$ along any direction $v \in\left\{L, v^{*}\right\}$, then

$$
\begin{aligned}
\operatorname{dim}(L)+1 & \leq \operatorname{LLI}\left(u^{*}\right), \\
\operatorname{dim}(L)+1 & \leq M I\left(u^{*}\right)+\operatorname{dim}\left(H^{0} \cap\left\{L, u^{*}\right\}\right), \\
M I\left(u^{*}\right)+\operatorname{dim}\left(H^{0} \cap\left\{L, u^{*}\right\}\right) & \leq L L I\left(u^{*}\right),
\end{aligned}
$$

or

$$
\operatorname{dim}(L)+1 \leq M I\left(u^{*}\right)+\operatorname{dim}\left(H^{0} \cap\left\{L, u^{*}\right\}\right) \leq L L I\left(u^{*}\right) .
$$

Proof. Suppose (2.2) does not hold, and let $H=H^{I} \oplus H^{D}$ as in (1.9) and (1.10). Then

$$
\operatorname{dim}(L)+1>\operatorname{LLI}\left(u^{*}\right) \equiv \operatorname{dim}\left(H^{D}\right) \quad \text { implies } \quad H^{I} \cap\left\{L, v^{*}\right\} \neq\{0\} .
$$

Thus $u^{*}$ is a local minimum point of $J$ along any direction in $H^{I} \cap\left\{L, v^{*}\right\}$ and this leads to a contradiction. Therefore (2.2) holds.

To prove (2.3), let $H=H^{-} \oplus H^{0} \oplus H^{+}$as in (1.6). If (2.3) is not true, then

$$
\operatorname{dim}\left(\left\{L, v^{*}\right\}\right)>\operatorname{dim}\left(H^{-}\right)+\operatorname{dim}\left(H^{0} \cap\left\{L, v^{*}\right\}\right)
$$

implies

$$
\left(H^{+} \oplus\left(H^{0} \cap\left\{L, v^{*}\right\}\right)_{H^{0}}^{\perp}\right) \cap\left\{L, v^{*}\right\} \neq\{0\},
$$

since

$$
H=H^{-} \oplus\left(H^{0} \cap\left\{L, v^{*}\right\}\right) \oplus H^{+} \oplus\left(H^{0} \cap\left\{L, v^{*}\right\}\right)_{H^{0}}^{\perp} .
$$

Choose any $v=v^{+}+v^{0} \in\left(H^{+} \oplus\left(H^{0} \cap\left\{L, v^{*}\right\}\right)_{H^{0}}^{\perp}\right) \cap\left\{L, v^{*}\right\}$ with $v \neq 0, v^{+} \in H^{+}$ and $v^{0} \in\left(H^{0} \cap\left\{L, v^{*}\right\}\right)_{H^{0}}^{\perp}$. Since $v^{+}=0$ will lead to

$$
v=v^{0} \in\left(H^{0} \cap\left\{L, v^{*}\right\}\right) \cap\left(\left(H^{0} \cap\left\{L, v^{*}\right\}\right)_{H^{0}}^{\perp}\right)=\{0\},
$$


which is a contradiction, we must have $v^{+} \neq 0$. Note that $H^{+}$and $H^{0}$ are mutually orthogonal invariant subspaces of the operator $J^{\prime \prime}\left(u^{*}\right)$. For such $v=v^{+}+v^{0} \in$ $\left\{L, v^{*}\right\}$, we have

$$
\left\langle J^{\prime \prime}\left(u^{*}\right)\left(v^{+}+v^{0}\right), v^{+}+v^{0}\right\rangle=\left\langle J^{\prime \prime}\left(u^{*}\right) v^{+}, v^{+}\right\rangle>0,
$$

i.e., $v$ is a strictly increasing direction of $J$ at $u^{*}$ in $\left\{L, v^{*}\right\}$, a contradiction to our assumption.

To verify (2.4), we note that

$$
\text { MI }\left(u^{*}\right)+\operatorname{dim}\left(H^{0} \cap\left\{L, v^{*}\right\}\right)>\operatorname{LLI}\left(u^{*}\right)
$$

implies

$$
H^{I} \cap\left(H^{-} \oplus\left(H^{0} \cap\left\{L, v^{*}\right\}\right)\right) \neq\{0\} .
$$

Let $w \in H^{I} \cap\left(H^{-} \oplus\left(H^{0} \cap\left\{L, v^{*}\right\}\right)\right)$ with $\|w\|=1$. Thus $w \in H^{I}$ is an increasing direction of $J$ at $u^{*}$. Write $w=w^{-}+w^{0}$ with $w^{-} \in H^{-}$and $w^{0} \in H^{0} \oplus\left\{L, v^{*}\right\}$. If $w^{-} \neq 0$, then $w$ is a strictly decreasing direction of $J$ at $u^{*}$. It leads to a contradiction. Thus we have $w^{-}=0$ which implies $w=w^{0} \in H^{I} \cap\left(H^{0} \cap\left\{L, v^{*}\right\}\right)$, i.e., $w$ is an increasing direction of $J$ at $u^{*}$ in $\left\{L, v^{*}\right\}$. Thus $J$ attains its local minimum at $u^{*}$ along $w \in\left\{L, v^{*}\right\}$, which contradicts our assumption again. Therefore (2.4) must be true. Combining (2.3) and (2.4), we obtain (2.5)).

Theorem 2.3. $M I\left(u^{*}\right)+\operatorname{dim}\left(H^{0} \cap H^{D}\right)=\operatorname{LLI}\left(u^{*}\right)$.

Proof. Note that

$$
H^{-} \oplus\left(H^{0} \cap H^{D}\right) \oplus\left(H^{0} \cap H^{D}\right)_{H^{0}}^{\perp} \oplus H^{+}=H=H^{I} \oplus H^{D} .
$$

First let us suppose that

$$
\text { MI }\left(u^{*}\right)+\operatorname{dim}\left(H^{0} \cap H^{D}\right)>\operatorname{LLI}\left(u^{*}\right) .
$$

Then we must have

$$
\left(H^{-} \oplus\left(H^{0} \cap H^{D}\right)\right) \cap H^{I} \neq\{0\} .
$$

For any $w \in\left(H^{-} \oplus\left(H^{0} \cap H^{D}\right)\right) \cap H^{I}$ with $w \neq 0$, we write $w=w^{-}+w^{0}$ where $w^{-} \in H^{-}$and $w^{0} \in H^{0} \cap H^{D}$. If $w^{-} \neq 0$, then $w$ is a strictly decreasing direction of $J$ at $u^{*}$, which will contradict the condition that $w \in H^{I}$. Thus we must have $w^{-}=0$, i.e., $w=w^{0} \in H^{0} \cap H^{D}$. It ends up with $0 \neq w \in H^{D} \cap H^{I}=\{0\}$. Therefore

$$
\text { MI }\left(u^{*}\right)+\operatorname{dim}\left(H^{0} \cap H^{D}\right) \leq \operatorname{LLI}\left(u^{*}\right) .
$$

Next let us suppose that

$$
\text { MI }\left(u^{*}\right)+\operatorname{dim}\left(H^{0} \cap H^{D}\right)<\operatorname{LLI}\left(u^{*}\right) .
$$

Then we must have

$$
\left(H^{+} \oplus\left(H^{0} \cap H^{D}\right)_{H^{0}}^{\perp}\right) \cap H^{D} \neq\{0\} .
$$

For any $w \in\left(H^{+} \oplus\left(H^{0} \cap H^{D}\right)_{H^{0}}^{\perp}\right) \cap H^{D}$ with $w \neq 0$, we write $w=w^{+}+w^{0}$ where $w^{+} \in H^{+}$and $w^{0} \in\left(H^{0} \cap H^{D}\right)_{H^{0}}^{\perp}$. Since $w^{0} \in H^{0}, w^{+} \neq 0$ will imply that $w=w^{+}+w^{0}$ is a strictly increasing direction; thus $w$ cannot be in $H^{D}$ at same time. Therefore we must have $w^{+}=0$. Then it leads to $w=w^{0} \in\left(H^{0} \cap H^{D}\right)_{H^{0}}^{\perp} \cap H^{D}=$ $\{0\}$. But we assume $w \neq 0$. Thus we must have

$$
\text { MI }\left(u^{*}\right)+\operatorname{dim}\left(H^{0} \cap H^{D}\right) \geq \operatorname{LLI}\left(u^{*}\right) .
$$


Combining the two inequalities, we conclude that

$$
\text { MI }\left(u^{*}\right)+\operatorname{dim}\left(H^{0} \cap H^{D}\right)=\operatorname{LLI}\left(u^{*}\right) .
$$

Combining the last two theorems, we have

$\operatorname{dim}(L)+1 \leq \mathrm{MI}\left(u^{*}\right)+\operatorname{dim}\left(H^{0} \cap\left\{L, v^{*}\right\}\right) \leq \mathrm{MI}\left(u^{*}\right)+\operatorname{dim}\left(H^{0} \cap H^{D}\right)=\operatorname{LLI}\left(u^{*}\right)$.

The inequality (2.4) displays the relation between the Morse index (MI) and the local linking index (LLI). It is clear that

$$
\operatorname{dim}(L)+1=\operatorname{MI}\left(u^{*}\right)+\operatorname{dim}\left(H^{0} \cap\left\{L, v^{*}\right\}\right)=\operatorname{LLI}\left(u^{*}\right),
$$

if $\left\{L, v^{*}\right\}^{\perp}$ contains no decreasing direction, i.e., $\left\{L, v^{*}\right\}^{\perp} \cap H^{D}=\{0\}$. But this condition is not easy to check. Thus next we establish the above equality with a condition that can be computationally checked.

Lemma 2.2. Let $p$ be a local peak selection of $J$ w.r.t. $L$ and continuous at $v^{*} \in S_{L^{\perp}}$. Assume $u^{*} \equiv p\left(v^{*}\right) \notin L$ and $v^{*}=\arg \min _{v \in S_{L^{\perp}}} J(p(v))$. Then

$$
\left(J^{\prime \prime}\left(u^{*}\right)\left(H^{-} \cap\left\{L, v^{*}\right\}^{\perp}\right)\right) \cap\left\{L, v^{*}\right\}^{\perp}=\{0\} .
$$

Proof. Since $u^{*}=p\left(v^{*}\right) \in\left\{L, v^{*}\right\}$ is a critical point of $J$ and also a local maximum point of $J$ in $\left\{L, v^{*}\right\}$, we have

$$
\left\langle J^{\prime \prime}\left(u^{*}\right) v, v\right\rangle \leq 0, \quad \forall v \in\left\{L, v^{*}\right\} .
$$

Suppose that the conclusion does not hold, i.e.,

$$
\left(J^{\prime \prime}\left(u^{*}\right)\left(H^{-} \cap\left\{L, v^{*}\right\}^{\perp}\right)\right) \cap\left\{L, v^{*}\right\}^{\perp} \neq\{0\} .
$$

Note that

$$
\left(J^{\prime \prime}\left(u^{*}\right)\left(H^{-} \cap\left\{L, v^{*}\right\}^{\perp}\right)\right) \cap\left\{L, v^{*}\right\}^{\perp}
$$

is a subspace. We can choose $w \in\left\{L, v^{*}\right\}^{\perp} \cap H^{-}$with $\|w\|=1$ s.t.

$$
J^{\prime \prime}\left(u^{*}\right)(w) \in\left(\left\{L, v^{*}\right\}^{\perp} \backslash\{0\}\right) .
$$

Since $H^{-}$is an invariant subspace of $J^{\prime \prime}\left(u^{*}\right)$, it implies that

$$
J^{\prime \prime}\left(u^{*}\right)(w) \in\left\{L, v^{*}\right\}^{\perp} \cap H^{-} \text {and } \quad J^{\prime \prime}\left(u^{*}\right)(w) \neq 0 .
$$

For any real number $s$ with $|s|$ small, we define

$$
v^{*}(s)=\frac{v^{*}+s w}{\left\|v^{*}+s w\right\|} \in \mathcal{N}\left(v^{*}\right) \cap S_{L^{\perp}} .
$$

Following the definition of the local peak selection, we can write

$$
p\left(v^{*}(s)\right)-u^{*}=p\left(v^{*}(s)\right)-p\left(v^{*}\right)=t^{*}(s) w+t_{s} v^{*}+v_{L}^{s}
$$

for some scalars $t^{*}(s), t_{s}$ and vector $v_{L}^{s} \in L$ where since $p$ is continuous at $v^{*}$ and $p\left(v^{*}\right) \notin L$, we have $t^{*}(s) \neq 0$ and $t^{*}(s) \rightarrow 0$ as $s \rightarrow 0$. By (2.8), taking (2.7) into account and with $J^{\prime \prime}\left(u^{*}\right)$ self-adjoint, we have

$$
\left\langle J^{\prime \prime}\left(u^{*}\right)\left(p\left(v^{*}(s)\right)-u^{*}\right),\left(p\left(v^{*}(s)\right)-u^{*}\right)\right\rangle<0 \quad \forall s \neq 0 \quad \text { and }|s| \text { is small. }
$$

Then for $|s|$ sufficiently small and $s \neq 0$, it follows that

$$
J\left(p\left(v^{*}(s)\right)\right)<J\left(u^{*}\right) \equiv J\left(p\left(v^{*}\right)\right) .
$$


On the other hand we have

$$
v^{*}=\arg \min _{v \in S_{L^{\perp}}} J(p(v))
$$

and $v^{*}(s) \in \mathcal{N}\left(v^{*}\right) \cap S_{L^{\perp}}$ or $J\left(p\left(v^{*}(s)\right)\right) \geq J\left(u^{*}\right)$, which leads to a contradiction. Thus (2.6) must hold.

It is clear that (2.6) implies that $u^{-} \notin\left\{L, v^{*}\right\}^{\perp}$ where $u^{-}$is an eigenfunction of $J^{\prime \prime}\left(u^{*}\right)$ w.r.t. a negative eigenvalue and (2.6) holds if $H^{-} \cap\left\{L, v^{*}\right\}=\{0\}$.

The proof of the following lemma follows a similar line to the proof of Lemma 2.1 in 22, but the conclusion is much stronger.

Lemma 2.3. Let $v^{*} \in S_{L^{\perp}}$. Assume that there exist a neighborhood $\mathcal{N}\left(v^{*}\right)$ of $v^{*}$ and a locally defined mapping $p: \mathcal{N}\left(v^{*}\right) \cap S_{L^{\perp}} \rightarrow H$ s.t. $p(v) \in\{L, v\} \forall v \in$ $\mathcal{N}\left(v^{*}\right) \cap S_{L^{\perp}}$. If $p$ is differentiable at $v^{*}$ and $u^{*}=p\left(v^{*}\right) \notin L$, then

$$
\begin{array}{cl}
w \in\left\{p^{\prime}\left(v^{*}\right)(w), L, v^{*}\right\} & \forall w \in\left\{L, v^{*}\right\}^{\perp}, \\
p^{\prime}\left(v^{*}\right)(w) \notin\left\{L, v^{*}\right\} & \forall w \in\left\{L, v^{*}\right\}^{\perp} \text { with } w \neq 0
\end{array}
$$

or

$$
p^{\prime}\left(v^{*}\right)\left(\left\{L, v^{*}\right\}^{\perp}\right) \oplus\left\{L, v^{*}\right\}=H .
$$

Proof. For any $w \in\left\{L, v^{*}\right\}^{\perp},\|w\|=1$, denote $v^{*}(s)=\frac{v^{*}+s w}{\left\|v^{*}+s w\right\|}$. Then there exists $s_{0}>0$ s.t. when $|s|<s_{0}$, we have $v^{*}(s) \in \mathcal{N}\left(v^{*}\right) \cap S_{L^{\perp}}$.

Consider the one-dimensional vector function $\alpha(s)=P_{L^{\perp}}\left(p\left(v^{*}(s)\right)\right)$, where $P_{L^{\perp}}$ is the projection onto $L^{\perp}$. Since $p$ is differentiable at $v^{*}$ and $v^{*}(s)$ smoothly depends on $s, \alpha$ is differentiable at 0 and

$$
\alpha^{\prime}(0)=P_{L^{\perp}}\left(p^{\prime}\left(v^{*}\right)\left(\frac{\partial v^{*}(s)}{\partial s}\right)\right)=P_{L^{\perp}}\left(p^{\prime}\left(v^{*}\right)(w)\right) .
$$

On the other hand, $p\left(v^{*}(s)\right) \in\left\{L, v^{*}(s)\right\}$, and we have $\alpha(s)=t_{s} v^{*}(s)$, where $t_{s}=\left\langle\alpha(s), v^{*}(s)\right\rangle$ is differentiable. Thus $\alpha^{\prime}(0)=t_{0}^{\prime} v^{*}+t_{0} w$, where due to our assumption that $u^{*}=p\left(v^{*}\right) \notin L$, we have $t_{0} \neq 0$. The two different expressions of $\alpha^{\prime}(0)$ imply

$$
P_{L^{\perp}}\left(p^{\prime}\left(v^{*}\right)(w)\right)=t_{s}^{\prime}(0) v^{*}+t_{0} w .
$$

Then it leads to $w \in\left\{p^{\prime}\left(v^{*}\right)(w), L, v^{*}\right\}$, i.e., (2.9) is verified.

Now if $w \in\left\{L, v^{*}\right\}^{\perp}$ with $w \neq 0$ and $p^{\prime}\left(v^{*}\right)(w) \in\left\{L, v^{*}\right\}$, (2.9) will lead to $w \in\left\{L, v^{*}\right\}$ and therefore $w=0$, a contradiction. Thus (2.10) holds. It is clear that (2.9) and (2.10) imply (2.11).

Lemma 2.4. Let $v^{*} \in S_{L^{\perp}}$. Assume that there exist a neighborhood $\mathcal{N}\left(v^{*}\right)$ of $v^{*}$ and a locally defined mapping $p: \mathcal{N}\left(v^{*}\right) \cap S_{L^{\perp}} \rightarrow H$ s.t. $p(v) \in\{L, v\} \forall v \in$ $\mathcal{N}\left(v^{*}\right) \cap S_{L^{\perp}}, p$ is differentiable at $v^{*}$ and $u^{*}=p\left(v^{*}\right) \notin L$ is a critical point of $J$. Denote

If $\operatorname{dim}\left(H_{0}^{-}\right)>\operatorname{dim} L+1$, then

or

$$
H_{0}^{-}=H^{-} \oplus\left(H^{0} \cap\left\{L, v^{*}\right\}\right) .
$$

$$
\begin{aligned}
p^{\prime}\left(v^{*}\right)\left(\left\{L, v^{*}\right\}^{\perp}\right) \cap H_{0}^{-} & \neq\{0\}, \\
p^{\prime}\left(v^{*}\right)\left(\left\{L, v^{*}\right\}^{\perp}\right) \cap\left(H^{0} \cap\left\{L, v^{*}\right\}\right) & =\{0\}
\end{aligned}
$$

$$
p^{\prime}\left(v^{*}\right)\left(\left\{L, v^{*}\right\}^{\perp}\right) \cap\left(H_{0}^{-} \backslash\left(H^{0} \cap\left\{L, v^{*}\right\}\right)\right) \neq\{0\} .
$$


Proof. Suppose that $k \equiv \operatorname{dim}(L)+1<\operatorname{dim}\left(H_{0}^{-}\right)$. By applying (2.11), there exit linearly independent vectors $e_{0}, e_{1}, \ldots, e_{k} \in H_{0}^{-}$that can be written as $e_{i}=g_{i}+f_{i}$ with $g_{i} \in p^{\prime}\left(v^{*}\right)\left(\left\{L, v^{*}\right\}^{\perp}\right)$ and $f_{i} \in\left\{L, v^{*}\right\}$. Here $f_{0}, f_{1}, \ldots, f_{k}$ have to be linearly dependent because $k=\operatorname{dim} L+1$. Thus we can find real numbers $a_{0}, a_{1}, \ldots, a_{k}$ s.t. $\sum_{i=0}^{k} a_{i}^{2} \neq 0$ and $\sum_{i=0}^{k} a_{i} f_{i}=0$. Therefore

$$
\sum_{i=0}^{k} a_{i} e_{i}=\sum_{i=0}^{k} a_{i} g_{i} \in p^{\prime}\left(v^{*}\right)\left(\left\{L, v^{*}\right\}^{\perp}\right) \cap H_{0}^{-} .
$$

Because $e_{0}, e_{1}, \ldots, e_{k}$ are linearly independent, $\sum_{i=0}^{k} a_{i} e_{i} \neq 0$. Thus, 2.13) holds. While (2.14) follows from (2.10) directly, (2.15) is a combination of (2.13) and (2.14).

Theorem 2.4. Let $v^{*} \in S_{L^{\perp}}$. Assume that $J$ has a local peak selection $p$ w.r.t. $L$ at $v^{*}$ s.t. $p$ is continuous at $v^{*}, u^{*} \equiv p\left(v^{*}\right) \notin L$ and $v^{*}=\arg \min _{v \in S_{L^{\perp}}} J(p(v))$. If either $H^{-} \cap\left\{L, v^{*}\right\}^{\perp}=\{0\}$ or $p$ is differentiable at $v^{*}$, then $u^{*}$ is a critical point with

$$
\operatorname{dim}(L)+1=M I\left(u^{*}\right)+\operatorname{dim}\left(H^{0} \cap\left\{L, v^{*}\right\}\right)
$$

where $H^{0}$ is the null space of $J^{\prime \prime}\left(u^{*}\right)$ as stated in (1.6)).

Proof. By Theorem [2.1, $u^{*} \equiv p\left(v^{*}\right)=t^{*} v^{*}+v_{L}^{*}$ for some $t^{*} \neq 0$ and $v_{L}^{*} \in L$ is a critical point of $J$; therefore, we only have to verify that equality (2.16) holds. Let $\mathcal{N}\left(v^{*}\right)$ be a neighborhood of $v^{*}$ in which the local peak selection $p$ is defined and let $H=H^{-} \oplus H^{0} \oplus H^{+}$as in (1.6). Denote

$$
H_{0}^{-}=H^{-} \oplus\left(H^{0} \cap\left\{L, v^{*}\right\}\right) .
$$

Suppose that (2.16) does not hold, by Theorem 2.2, we have

$$
\operatorname{dim}\left(H_{0}^{-}\right)>\operatorname{dim}(L)+1 \text { implies }\left\{L, v^{*}\right\}^{\perp} \cap H_{0}^{-} \neq\{0\} .
$$

For any $w \in\left\{L, v^{*}\right\}^{\perp} \cap H_{0}^{-}$with $w \neq 0$, we can write $w=w^{-}+w^{0}$ with $w^{-} \in H^{-}$ and $w^{0} \in H^{0} \cap\left\{L, v^{*}\right\}$. Taking the inner product of $w$ with $w_{0}$ gives $w_{0}=0$. Thus

$$
\left(\left\{L, v^{*}\right\}^{\perp} \cap H_{0}^{-}\right)=\left(\left\{L, v^{*}\right\}^{\perp} \cap H^{-}\right) \neq\{0\} .
$$

Therefore if $\left\{L, v^{*}\right\}^{\perp} \cap H^{-}=\{0\}$, (2.16) must hold.

If instead we assume that $p$ is differentiable at $v^{*}$, then 2.17) and Lemma 2.4 imply that

$$
p^{\prime}\left(\left\{L, v^{*}\right\}^{\perp}\right) \cap\left(H_{0}^{-} \backslash\left(H^{0} \cap\left\{L, v^{*}\right\}\right)\right) \neq\{0\} .
$$

Choose any $w \in\left\{L, v^{*}\right\}^{\perp},\|w\|=1$, s.t. $p^{\prime}\left(v^{*}\right)(w) \in\left(H_{0}^{-} \backslash\left(H^{0} \cap\left\{L, v^{*}\right\}\right)\right)$. Around $u^{*}=p\left(v^{*}\right)$, we have the second order Taylor expansion

$$
J(u)=J\left(u^{*}\right)+\frac{1}{2}\left\langle J^{\prime \prime}\left(u^{*}\right)\left(u-u^{*}\right), u-u^{*}\right\rangle+o\left(\left\|u-u^{*}\right\|^{2}\right) .
$$

Denote $v^{*}(s)=\frac{v^{*}+s w}{\left\|v^{*}+s w\right\|}$. We have $v^{*}(s) \in \mathcal{N}\left(v^{*}\right) \cap S_{L^{\perp}}$ for $|s|$ small and $\left.\frac{d v^{*}(s)}{d s}\right|_{s=0}$ $=w$. Thus it follows that

$$
u_{s} \equiv p\left(v^{*}(s)\right)=u^{*}+s p^{\prime}\left(v^{*}\right)(w)+o(|s|) .
$$


Combining the above two estimates (2.18) and (2.19), we obtain

$$
J\left(p\left(v^{*}(s)\right)\right)=J\left(u^{*}\right)+\frac{1}{2} s^{2}\left\langle J^{\prime \prime}\left(u^{*}\right)\left(p^{\prime}\left(v^{*}\right)(w)\right), p^{\prime}\left(v^{*}\right)(w)\right\rangle+o\left(s^{2}\right)<J\left(u^{*}\right),
$$

where the last strict inequality holds for $|s|$ sufficiently small because

$p^{\prime}\left(v^{*}\right)(w) \in\left(H_{0}^{-} \backslash\left(H^{0} \cap\left\{L, v^{*}\right\}\right)\right) \quad$ implies $\left\langle J^{\prime \prime}\left(u^{*}\right)\left(p^{\prime}\left(v^{*}\right)(w)\right), p^{\prime}\left(v^{*}\right)(w)\right\rangle<0$.

Since $v^{*}(s) \in \mathcal{N}\left(v^{*}\right) \cap S_{L^{\perp}}$ and $u^{*}=p\left(v^{*}\right)$, the above violates the assumption that $v^{*}$ is a local minimum point of $J \circ p$ on $S_{L^{\perp}}$. Therefore (2.16) is verified.

Remark 2.1. The result in the last theorem is multi-fold. First, it provides a method to evaluate the Morse index of a saddle point without actually finding $\operatorname{dim}\left(H^{-}\right)$, a very expensive job. Secondly, it indicates that $\operatorname{dim}(L)+1$ is better than MI in measuring local instability for a degenerate saddle point $u^{*}$. It also provides a guideline in numerical computation. It implies that to have a stable convergence in computing a critical point of $\mathrm{MI}=n$, the support $L$ must contain at least $n-1$ critical points at lower critical level.

Two questions still remain: how to determine $\operatorname{dim}\left(H^{0} \cap\left\{L, v^{*}\right\}\right)$ and how to check if $p$ is differentiable at $v^{*}$ ? Since it is usually very expensive to find $H^{0}$, we point out that one does not have to find $H^{0}$ to determine $\operatorname{dim}\left(H^{0} \cap\left\{L, v^{*}\right\}\right)$. To see this, let $L=\left\{w_{1}, \ldots, w_{n}\right\}$ where $w_{1}, \ldots, w_{n}$ are linearly independent and define the quadratic function

$$
\begin{aligned}
& Q\left(t_{0}, t_{1}, \ldots, t_{n}\right) \\
& \quad \equiv \frac{1}{2}\left\langle J^{\prime \prime}\left(u^{*}\right)\left(t_{0} v^{*}+t_{1} w_{1}+\cdots+t_{n} w_{n}\right),\left(t_{0} v^{*}+t_{1} w_{1}+\cdots+t_{n} w_{n}\right)\right\rangle .
\end{aligned}
$$

We have

$$
Q\left(t_{0}, t_{1}, \ldots, t_{n}\right)=\frac{1}{2}\left(t_{0}, t_{1}, \ldots, t_{n}\right) Q^{\prime \prime}\left(t_{0}, t_{1}, \ldots, t_{n}\right)^{T}
$$

where $Q^{\prime \prime}$ is the $(n+1) \times(n+1)$ matrix

$$
Q^{\prime \prime}=\left[\begin{array}{cccc}
\left\langle J^{\prime \prime}\left(u^{*}\right) v^{*}, v^{*}\right\rangle & \left\langle J^{\prime \prime}\left(u^{*}\right) v^{*}, w_{1}\right\rangle & \cdots & \left\langle J^{\prime \prime}\left(u^{*}\right) v^{*}, w_{n}\right\rangle \\
\left\langle J^{\prime \prime}\left(u^{*}\right) w_{1}, v^{*}\right\rangle & \left\langle J^{\prime \prime}\left(u^{*}\right) w_{1}, w_{1}\right\rangle & \cdots & \left\langle J^{\prime \prime}\left(u^{*}\right) w_{1}, w_{n}\right\rangle \\
\cdots & \cdots & \cdots & \cdots \\
\left\langle J^{\prime \prime}\left(u^{*}\right) w_{n}, v^{*}\right\rangle & \left\langle J^{\prime \prime}\left(u^{*}\right) w_{n}, w_{1}\right\rangle & \cdots & \left\langle J^{\prime \prime}\left(u^{*}\right) w_{n}, w_{n}\right\rangle
\end{array}\right] .
$$

Let us observe that $\left(t_{0} v^{*}+t_{1} w_{1}+\cdots+t_{n} w_{n}\right) \in H^{0} \cap\left\{L, v^{*}\right\}$ implies $\left(t_{0}, t_{1}, \ldots, t_{n}\right) \in$ $\operatorname{ker}\left(Q^{\prime \prime}\right)$, i.e.,

$$
\left(H^{0} \cap\left\{L, v^{*}\right\}\right) \subset\left\{t_{0} v^{*}+t_{1} w_{1}+\cdots+t_{n} w_{n}:\left(t_{0}, t_{1}, \ldots, t_{n}\right) \in \operatorname{ker}\left\{Q^{\prime \prime}\right\}\right\} .
$$

Thus if $\operatorname{ker}\left\{Q^{\prime \prime}\right\}=\{0\}$ or $\left|Q^{\prime \prime}\right| \neq 0$, then $\operatorname{dim}\left(H^{0} \cap\left\{L, v^{*}\right\}\right)=0$ even if $H^{0} \neq$ $\{0\}$. When $\operatorname{ker}\left\{Q^{\prime \prime}\right\} \neq\{0\}$, let $\left\{T_{1}, \ldots, T_{r}\right\}$ be a basis of $\operatorname{ker}\left\{Q^{\prime \prime}\right\}$ where $T_{i}=$ $\left(T_{i}^{0}, T_{i}^{1}, \ldots, T_{i}^{n}\right) \in R^{n+1}$. Denote

$$
z_{i}=T_{i}^{0} J^{\prime \prime}\left(u^{*}\right) v^{*}+\sum_{j=1}^{n} T_{i}^{j} J^{\prime \prime}\left(u^{*}\right) w_{j}, \quad i=1, \ldots, r
$$

and solve the linear homogenous system

$$
t_{1} z_{1}+\cdots+t_{r} z_{r}=0
$$

for $\left(t_{1}, \ldots, t_{r}\right) \in R^{r}$. Then $\operatorname{dim}\left(H^{0} \cap\left\{L, v^{*}\right\}\right)=$ the dimension of the solution space to (2.22). 
The above analysis together with Theorem 2.4 can be summarized as

Theorem 2.5. Let $v^{*} \in S_{L^{\perp}}$. Assume $J$ has a local peak selection $p$ w.r.t. $L$ at $v^{*}$ s.t. $p$ is continuous at $v^{*}, u^{*} \equiv p\left(v^{*}\right) \notin L$ and $v^{*}=\arg \min _{v \in S_{L^{\perp}}} J(p(v))$. If either $H^{-} \cap\left\{L, v^{*}\right\}^{\perp}=\{0\}$ or $p$ is differentiable at $v^{*}$, then $u^{*}$ is a critical point with

(2.23) $0 \leq \operatorname{dim}(L)+1-M I\left(u^{*}\right)=\operatorname{dim}($ Solution Space to 2.22$\left.)\right) \leq \operatorname{dim}\left(\operatorname{ker}\left\{Q^{\prime \prime}\right\}\right)$

where the matrix $Q^{\prime \prime}$ is defined in (2.21). In addition, if the matrix $Q^{\prime \prime}$ is nonsingular, then

$$
\operatorname{dim}(L)+1=M I\left(u^{*}\right) .
$$

Since $u^{*}$ is a critical point of $J$ and also a local maximum point of $J$ in $\left\{L, v^{*}\right\}$, we have

$$
Q\left(t_{0}, t_{1}, \ldots, t_{n}\right) \leq 0 \quad \forall\left(t_{0}, t_{1}, \ldots, t_{n}\right) \text { close to }(0, \ldots, 0) .
$$

But $Q\left(t_{0}, t_{1}, \ldots, t_{n}\right)$ is quadratic and $Q(0, \ldots, 0)=0$. It follows that

$$
Q\left(t_{0}, t_{1}, \ldots, t_{n}\right) \leq 0 \quad \forall\left(t_{0}, t_{1}, \ldots, t_{n}\right) \in \mathbb{R}^{n+1},
$$

i.e., $Q^{\prime \prime}$ has to be semi-negative definite. Thus

$$
\left|Q^{\prime \prime}\right| \neq 0
$$

will be enough to ensure that $Q^{\prime \prime}$ is negative definite and $\operatorname{dim}\left(H^{0} \cap\left\{L, v^{*}\right\}\right)=0$. For $L=\{0\}$, (2.24) becomes

$$
Q^{\prime \prime}=\left\langle J^{\prime \prime}\left(u^{*}\right) v^{*}, v^{*}\right\rangle<0
$$

and for $L=\left\{w_{1}\right\}$, (2.24) has to be

$$
\left|Q^{\prime \prime}\right|=\left|\begin{array}{ll}
\left\langle J^{\prime \prime}\left(u^{*}\right) v^{*}, v^{*}\right\rangle & \left\langle J^{\prime \prime}\left(u^{*}\right) v^{*}, w_{1}\right\rangle \\
\left\langle J^{\prime \prime}\left(u^{*}\right) w_{1}, v^{*}\right\rangle & \left\langle J^{\prime \prime}\left(u^{*}\right) w_{1}, w_{1}\right\rangle
\end{array}\right|>0
$$

which implies $\left\langle J^{\prime \prime}\left(u^{*}\right) v^{*}, v^{*}\right\rangle<0$ and $\left\langle J^{\prime \prime}\left(u^{*}\right) w_{1}, w_{1}\right\rangle<0$. To see this, first we note that $\left|Q^{\prime \prime}\right|<0$ implies that $(0,0)$ is a saddle point of $Q\left(t_{0}, t_{1}\right)$. Thus the $2 \times 2$ matrix $Q^{\prime \prime}$ has a positive and a negative eigenvalue. But this is impossible, since $Q^{\prime \prime}$ has to be semi-negative definite. Therefore it must be that $\left|Q^{\prime \prime}\right|>0$. Next we note that $J^{\prime \prime}\left(u^{*}\right)$ is self-adjoint, $J$ attains its local maximum in $\left\{w_{1}, v^{*}\right\}$ at $u^{*}$ and $J^{\prime}\left(u^{*}\right)=0$; thus $\left\langle J^{\prime \prime}\left(u^{*}\right) v^{*}, v^{*}\right\rangle \leq 0$ and $\left\langle J^{\prime \prime}\left(u^{*}\right) w_{1}, w_{1}\right\rangle \leq 0$. Then $\left|Q^{\prime \prime}\right|>0$ implies that $\left\langle J^{\prime \prime}\left(u^{*}\right) v^{*}, v^{*}\right\rangle<0$ and $\left\langle J^{\prime \prime}\left(u^{*}\right) w_{1}, w_{1}\right\rangle<0$.

Note that $Q^{\prime \prime}$ is a restriction of $J^{\prime \prime}\left(u^{*}\right)$ to the finite-dimensional space $\left\{L, v^{*}\right\}$ in some sense. When the infinite-dimensional linear operator $J^{\prime \prime}\left(u^{*}\right)$ is singular, i.e., $H^{0} \neq\{0\}$, the $(n+1) \times(n+1)$ matrix $Q^{\prime \prime}$ can still be nonsingular.

In numerical computation, at each iteration, we numerically compute $\left|Q^{\prime \prime}\right|$ at an approximation solution $u^{k}$. Numerous numerical examples have been carried out by us. In all the examples including many degenerate cases, the $\left|Q^{\prime \prime}\right|$ are bounded away from zero.

Next let us study how to check if $p$ is differentiable at $v^{*}$. This is very difficult for two reasons; first $p$ has no explicit formula and secondly a limit of a sequence of local maximum points of $J$ is not necessarily a local maximum point of $J$, i.e., the graph of $p$ may be not closed. Research to solve this problem has inspired us to develop a new approach which is already beyond the scope of a minimax principle. More profound analysis is required. Details will be reported in a companion paper 36. Here we briefly describe the idea. In [36], the local peak selection $p$ is generalized to satisfy an orthogonal condition, i.e., for $v \in S_{L^{\perp}}, p(v) \in\{L, v\}$ s.t. $J^{\prime}(p(v)) \perp\{L, v\}$, 
called a local orthogonal selection. It is clear that if $p$ is a local peak selection, then $p(v) \in\{L, v\}$ and $J^{\prime}(p(v)) \perp\{L, v\}$, i.e., $p$ is also a local orthogonal selection. For such a defined $p$, it is proved in [36] that if

$$
v^{*}=\arg \min _{v \in S_{L} \perp \cap \mathcal{N}\left(v^{*}\right)} J(p(v)),
$$

$u^{*}=p\left(v^{*}\right) \notin L$ and $p$ is continuous at $v^{*}$, then $u^{*}$ is a critical point of $J$. However, since such a saddle point is in general not a minimax solution, instability analysis will be much more complicated. For such a local orthogonal selection $p$, from the implicit function theorem if the matrix $Q^{\prime \prime}$ in (2.21) is nonsingular, then $p$ is differentiable near $v^{*}$. Then an interesting question can be asked if in addition $u^{*}=p\left(v^{*}\right)$ happens to be a local maximum point of $J$ in $\left\{L, v^{*}\right\}$ : will such a differentiable local orthogonal selection $p$ become a differentiable local peak selection near $v^{*}$ ? The answer is yes.

Theorem 2.6. Let $L$ be a finite-dimensional subspace of a Hilbert space $H$, let $v^{*} \in S_{L^{\perp}}$ and let $p$ be a local orthogonal selection of $J$ w.r.t. $L$ at $v^{*}$ s.t. $u^{*}=$ $p\left(v^{*}\right) \notin L$ and the matrix $Q^{\prime \prime}$ in (2.21) is invertible. Assume that

$$
v^{*}=\arg \min _{v \in S_{L} \perp \cap \mathcal{N}\left(v^{*}\right)} J(p(v))
$$

where $\mathcal{N}\left(v^{*}\right)$ is a neighborhood of $v^{*}$ s.t. the local orthogonal selection $p$ is defined in $S_{L^{\perp}} \cap \mathcal{N}\left(v^{*}\right)$ and where $u^{*}=p\left(v^{*}\right)$ is a local maximum point of $J$ in $\left\{L, v^{*}\right\}$. Then

(1) $\quad p$ is a differentiable local peak selection near $v^{*}$,

(2) $u^{*}=p\left(v^{*}\right)$ is a strict local maximum point of $J$ in $\left\{L, v^{*}\right\}$,

(3) $u^{*}=p\left(v^{*}\right)$ is a critical point of $J$ with $\operatorname{dim}(L)+1=M I\left(u^{*}\right)$.

Proof. Let $L=\left\{w_{1}, w_{2}, \ldots, w_{n}\right\}$. We only have to show that under the conditions, the local orthogonal selection $p$ is differentiable near $v^{*}$ and there exists $r>0$ s.t. for $v \in S_{L^{\perp}} \cap \mathcal{N}\left(v^{*}\right)$ with $\left\|v-v^{*}\right\|<r, u=p(v)$ is also a local maximum point of $J$ in $\{L, v\}$.

First note that when $u^{*}=p\left(v^{*}\right)$ is a local maximum point of $J$ in $\left\{L, v^{*}\right\}$ and the matrix $Q^{\prime \prime}$ in (2.21) is invertible, $Q^{\prime \prime}$ has to be negative definite and therefore $u^{*}=p\left(v^{*}\right)$ is a strict local maximum point of $J$ in $\left\{L, v^{*}\right\}$. Next observe that from its definition [36], the local orthogonal selection $u=p(v)=t_{0} v+t_{1} w_{1}+\cdots+t_{n} w_{n}$ is solved from the system

$$
\begin{aligned}
F_{0}\left(v, t_{0}, t_{1}, \ldots, t_{n}\right) \equiv & \left\langle J^{\prime}\left(t_{0} v+t_{1} w_{1}+\cdots+t_{n} w_{n}\right), v\right\rangle=0, \\
F_{1}\left(v, t_{0}, t_{1}, \ldots, t_{n}\right) \equiv & \left\langle J^{\prime}\left(t_{0} v+t_{1} w_{1}+\cdots+t_{n} w_{n}\right), w_{1}\right\rangle=0, \\
\cdots & \cdots \\
F_{n}\left(v, t_{0}, t_{1}, \ldots, t_{n}\right) \equiv & \left\langle J^{\prime}\left(t_{0} v+t_{1} w_{1}+\cdots+t_{n} w_{n}\right), w_{n}\right\rangle=0
\end{aligned}
$$

for each given $v \in S_{L^{\perp}} \cap \mathcal{N}\left(v^{*}\right)$. The Jacobian matrix

$$
\left.\frac{\partial\left(F_{0}, F_{1}, \ldots, F_{n}\right)}{\partial\left(t_{0}, t_{1}, \ldots, t_{n}\right)}\right|_{\left(v, t_{0}, t_{1}, \ldots, t_{n}\right)=\left(v^{*}, t_{0}^{*}, t_{1}^{*}, \ldots, t_{n}^{*}\right)}=Q^{\prime \prime}
$$

as in (2.21) where $u^{*}=t_{0}^{*} v^{*}+t_{1}^{*} w_{1}+\cdots+t_{n}^{*} w_{n}$. By the implicit function theorem, when the matrix $Q^{\prime \prime}$ is invertible, there is a neighborhood $\mathcal{N}_{0}\left(v^{*}\right)$ of $v^{*}$, s.t. the local orthogonal selection $p$ is uniquely defined and differentiable for each $v \in S_{L^{\perp}} \cap$ $\mathcal{N}_{0}\left(v^{*}\right)$. 
Suppose that such a local orthogonal selection $p$ is not a local peak selection. Then for any $r>0$ there exists $v \in S_{L^{\perp}} \cap \mathcal{N}\left(v^{*}\right)$ with $\left\|v-v^{*}\right\|<r$ s.t. for any $\delta>0$ there is $w \in\{L, v\}$ with $\|w-u\|<\delta$ and $J(w)>J(u)$ where $u=p(v)$. Under the conditions, $u^{*}=p\left(v^{*}\right)$ is a strict local maximum point of $J$ in $\left\{L, v^{*}\right\}$. Let $\delta_{0}>0$ be s.t.

$$
J\left(u^{*}\right)>J(w) \quad \forall w \in\left\{L, v^{*}\right\} \quad \text { with } \quad 0<\left\|w-u^{*}\right\|<\delta_{0} .
$$

For any $0<\delta<\delta_{0}$, since $\{w \in\{L, v\}:\|w-p(v)\|=\delta\}$ is a compact set, we denote

$$
J_{\delta}(v)=\max \{J(w): w \in\{L, v\},\|w-p(v)\|=\delta\} .
$$

Let $\epsilon=\frac{1}{4}\left(J\left(u^{*}\right)-J_{\delta}\left(v^{*}\right)\right)>0$. By the continuities of $J$ and $p$ at $v^{*}$, there exists $r_{0}>0$ s.t. for any $r$ with $0<r<r_{0}$, when $v \in S_{L^{\perp}}$ with $\left\|v-v^{*}\right\|<r, p(v)$ is defined and

$$
J_{\delta}\left(v^{*}\right)-\epsilon<J_{\delta}(v)<J_{\delta}\left(v^{*}\right)+\epsilon \text { and thus } \quad J\left(u^{*}\right)>J_{\delta}(v) .
$$

Since $v^{*}=\arg \min _{v \in S_{L} \perp \cap \mathcal{N}\left(v^{*}\right)} J(p(v))$, if $u=p(v)$ is not a local maximum point of $J$ in $\{L, v\}$, there exists $w_{\delta} \in\{L, v\}$ with $\left\|w_{\delta}-p(v)\right\|<\delta$ s.t.

$$
J\left(w_{\delta}\right)>J(u) \geq J\left(u^{*}\right) .
$$

Next we note that $J$ is continuous, and it attains its maximum on the compact set $\{w \in\{L, v\}:\|w-u\| \leq \delta\}$ at, say, $w_{v}$. We have

$$
J\left(w_{v}\right) \geq J\left(w_{\delta}\right)>J(u) \geq J\left(u^{*}\right)>J_{\delta}(v) .
$$

Thus $w_{v} \in\{L, v\}$ with $\left\|w_{v}-u\right\|<\delta$ and $w_{v} \neq u$. Therefore $J^{\prime}\left(w_{v}\right) \perp\{L, v\}$ or $w_{v} \in P(v)$, i.e., both $w_{v}$ and $u=p(v)$ are in the local orthogonal mapping $P(v)$. Since this holds for any $0<\delta<\delta_{0}$ and $0<r<r_{0}$, it violates the uniqueness conclusion of the implicit function theorem. Thus $p$ is a differentiable local peak selection of $J$ w.r.t. $L$ near $v^{*}$, and $u^{*}=p\left(v^{*}\right)$ is a critical point of $J$ as proved in [36. Finally, by $\left|Q^{\prime \prime}\right| \neq 0$ and Theorem 2.5 we conclude that $\operatorname{dim}(L)+1=$ MI $\left(u^{*}\right)$.

\section{Instability RELATIVe to A REFERENCE CRITICAL POINT}

The above definition of MMI can be viewed as an instability index of a saddle point $u^{*}$ relative to a local minimum critical point. This idea can be extended to define a local instability index of a saddle point $u^{*}$ relative to a given reference critical point $u^{0}$, either a trivial or nontrivial one for which information on local structure is available. This idea is particularly useful when a local minimum of $J$ does not exist or when $\operatorname{MI}\left(u^{*}\right)=+\infty$ for every saddle point $u^{*}$ of $J$. In this case the Morse indices of two critical points cannot tell the difference in their local instabilities, while our MMI can be used to measure a relative local instability between those two critical points.

Definition 3.1. Let $u^{0}$ be a given reference critical point and let $L^{0}$ be a closed maximum subspace of $H$ consisting of decreasing directions of $J$ at $u^{0}$. Let $L$ be a finite-dimensional subspace of $H$, consisting of only increasing directions of $J$ at $u^{0}$ and write $L^{1}=L^{0} \oplus L$. If $v^{1} \in S_{L^{1 \perp}}$ and if there is a local peak selection $p_{L^{1}}$ of $J$ w.r.t. $L^{1}$ near $v^{1}$ s.t. $p_{L^{1}}$ is continuous at $v^{1}, u^{1}=p_{L^{1}}\left(v^{1}\right) \notin L^{1}$ and

$$
v^{1}=\arg \min _{v \in S_{L^{1}}} J\left(p_{L^{1}}(v)\right)
$$


then the number $\operatorname{dim}(L)+1$ is called the local minimax index of the saddle point $u^{1}$ relative to the reference critical point $u^{0}$.

Similar to Definition 2.3 one can use minimization or maximization to make the definition unique. We focus on the instability information that the number $\operatorname{dim}(L)+$ 1 provides. There are several ways to define $L^{0}$ depending on the information available on the local structure of the reference critical point $u^{0}$.

(1) If $u^{0}$ is a minimax solution with a support $L_{0}$, then $L^{0}=L_{0} \oplus\left\{v^{0}\right\}$ where $u^{0}=p_{L_{0}}\left(v^{0}\right)$ for some local peak selection $p_{L_{0}}$ of $J$ w.r.t. $L_{0}$. By Lemma 2.1 $\left\{L_{0}, v^{0}\right\}$ is maximum.

(2) If the orthogonal spectral decomposition $H=H^{-} \oplus H^{0} \oplus H^{+}$as in (1.6) is available at $u^{0}$, where $H^{0}$ is finite-dimensional, then $L^{0}=H^{-} \oplus H^{0 D}$ where $H^{0 D}$ a maximum subspace of $H^{0}$ consisting of decreasing directions of $J$ at $u^{0}$. It is clear that such a subspace $L^{0}$ is maximum.

(3) If $u^{0}$ is a local linking with $H=H^{D} \oplus H^{I}$ as in (1.9) and (1.10), then simply $L^{0}=H^{D}$ is maximum.

In all the above three cases, $L^{0}$ and $\left\{L^{1}, v^{1}\right\}$ are, respectively, the decreasing direction subspaces of $J$ at $u^{0}=p_{L_{0}}\left(v^{0}\right)$ and $u^{1}=p_{L_{1}}\left(v^{1}\right)$, and $L^{0} \subset L^{1}$ with

$$
\operatorname{dim}\left(L^{1}\right)+1=\operatorname{dim}\left(L^{0}\right)+\operatorname{dim}(L)+1 .
$$

Since $L^{0}$ consists of decreasing directions common to both $u^{0}$ and $u^{1}$, it is natural to use the difference $\operatorname{dim}(L)+1$ to measure the local instability of $u^{1}$ relative to $u^{0}$, in particular, when $\operatorname{dim}\left(L^{0}\right)=\infty$ and therefore $\operatorname{dim}\left(L^{1}\right)=\infty$.

As in the notation in Definition 3.1, if $L^{0}$ is finite-dimensional and $H=H_{0}^{-} \oplus$ $H_{0}^{0} \oplus H_{0}^{+}$and $H_{1}^{-} \oplus H_{1}^{0} \oplus H_{1}^{+}$are, respectively, the orthogonal spectral decompositions of $H$ at $u^{0}$ and $u^{1}$, then we can prove the following theorems.

Theorem 3.1.

$$
\operatorname{dim}(L)+1 \leq M I\left(u^{1}\right)+\operatorname{dim}\left(H_{1}^{0} \cap\left\{L^{1}, v^{1}\right\}\right)-\operatorname{dim}\left(L^{0}\right) .
$$

Proof. Similar to that of Theorem 2.2 .

Theorem 3.2. If the local peak selection $p_{L^{1}}$ near $v^{1}$ is differentiable at $v^{1}$, respectively, then

$$
\operatorname{dim}(L)+1=M I\left(u^{1}\right)+\operatorname{dim}\left(H_{1}^{0} \cap\left\{L^{1}, v^{1}\right\}\right)-\operatorname{dim}\left(L^{0}\right) .
$$

Proof. Similar to that of Theorem 2.4.

Theorem 3.3. If the local peak selections $p_{L_{0}}$ near $v^{0}$ and $p_{L^{1}}$ near $v^{1}$ are differentiable at $v^{0}$ and $v^{1}$, respectively, then

$$
\operatorname{dim}(L)+1=M I\left(u^{1}\right)-M I\left(u^{0}\right)+\operatorname{dim}\left(H_{1}^{0} \cap\left\{L^{1}, v^{1}\right\}\right)-\operatorname{dim}\left(H_{0}^{0} \cap\left\{L_{0}, v^{0}\right\}\right) .
$$

Proof. Similar to that of Theorem 2.4.

\section{Applications}

Consider a nonlinear functional

$$
J(u)=\frac{1}{2}\langle A u, u\rangle-\int_{\Omega} F(x, u(x)) d x, \quad \forall u \in H
$$

where $\Omega$ is a bounded open domain in $\mathbb{R}^{n}$ with smooth boundary $\partial \Omega$ and $H=H(\Omega)$ is a Hilbert space of functions defined on $\Omega, A: H \rightarrow H$ is a bounded self-adjoint 
linear operator with the orthogonal spectral decomposition $H=H^{-} \oplus H^{0} \oplus H^{+}$ as stated in (1.6) and $F(x, \xi)=\int_{0}^{\xi} f(x, t) d t$. The function $f(x, \xi)$ satisfies some standard regularity and growth hypothesis s.t. $J$ is $C^{2}$ and has a local minimum at $u=0$. In addition, we assume that

$$
f_{\xi}^{\prime}(x, t \xi)>\frac{f(x, t \xi)}{t \xi} \quad \forall x \in \Omega, t>0, \quad \xi \neq 0 .
$$

For all numerical examples computed in 20] and 21], condition (H) is satisfied. If $u^{*} \in H$ is a saddle point of $J$, let $u=\frac{u^{*}}{\left\|u^{*}\right\|}$ and $t_{u}=\left\|u^{*}\right\|>0$. For all $t>0$, we have

and

$$
J(t u)=\frac{t^{2}}{2}\langle A u, u\rangle-\int_{\Omega} F(x, t u(x)) d x
$$

or

$$
\left.\frac{d J(t u)}{d t}\right|_{t=t_{u}}=t_{u}\langle A u, u\rangle-\int_{\Omega} f\left(x, t_{u} u(x)\right) u(x) d x=0
$$

$$
\langle A u, u\rangle=\int_{\Omega} \frac{f\left(x, t_{u} u(x)\right)}{t_{u} u(x)} u^{2}(x) d x .
$$

Condition $(\mathrm{H})$ implies that $\frac{f(x, t u(x))}{t u(x)}$ is monotone in $t>0$; therefore, such a $t_{u}>0$ is unique and satisfies

$$
\begin{aligned}
\left.\frac{d^{2} J(t u)}{d t^{2}}\right|_{t=t_{u}} & =\left\langle J^{\prime \prime}\left(t_{u} u\right) u, u\right\rangle=\langle A u, u\rangle-\int_{\Omega} f_{\xi}^{\prime}\left(x, t_{u} u(x)\right) u^{2}(x) d x \\
& <\langle A u, u\rangle-\int_{\Omega} \frac{f\left(x, t_{u} u(x)\right)}{t_{u} u(x)} u^{2}(x) d x=0
\end{aligned}
$$

That is, inequality (2.25) is satisfied. Thus $u^{*}$ must be a local maximum point of $J$ in the direction of $u$. We obtain

Proposition 4.1. Let condition $(\underline{\mathrm{H}})$ be satisfied and $u=0$ be a local minimum point of $J$. If $u^{*}=t_{u} u=\arg \min _{v \in S_{H}} J(p(v))$ is a nonzero critical point of $J$ with $\|u\|=1$ and $t_{u}>0$, then the local peak selection $p: v \in S_{H} \rightarrow t_{v} v p$ w.r.t. $L=\{0\}$ is differentiable at $u$ and, consequently, $u^{*}$ is not in the nullspace of $J^{\prime \prime}\left(u^{*}\right)$ and $\operatorname{MI}\left(u^{*}\right)=1$.

For superlinear elliptic equations studied in 20] and [21], we have

$$
\langle A u, u\rangle=\int_{\Omega}|\nabla u(x)|^{2} d x .
$$

Thus $H^{-}=H^{0}=\{0\}$. We first let $L=\{0\}$ and find a minimax solution $u^{*}$ by our local minimax method. From our analysis above we have $M I\left(u^{*}\right)=\operatorname{dim}(L)+1=1$ even if $u^{*}$ is degenerate.

Next let $w_{1}=u^{*}$, set $L=\left\{w_{1}\right\}$ and write $w_{2}=t^{*} v^{*}+t_{1}^{*} w_{1}$. Then (2.26) becomes

$$
\begin{array}{r}
\left(\left\langle A v^{*}, v^{*}\right\rangle-\int_{\Omega} f_{\xi}^{\prime}\left(x, w_{2}(x)\right) v^{* 2}(x) d x\right)\left(\left\langle A w_{1}, w_{1}\right\rangle-\int_{\Omega} f_{\xi}^{\prime}\left(x, w_{2}(x)\right) w_{1}^{2}(x) d x\right) \\
-\left(\left\langle A v^{*}, w_{1}\right\rangle-\int_{\Omega} f_{\xi}^{\prime}\left(x, w_{2}(x)\right) v^{*}(x) w_{1}(x) d x\right)^{2}>0 .
\end{array}
$$


This inequality has been numerically checked to be satisfied for each saddle point $w_{2}=\arg \min _{v \in S_{L \perp}} J(p(v))$ to every superlinear elliptic equation computed in 20, 21, which concludes $\operatorname{MI}\left(w_{2}\right)=2$. The inequality (2.21) corresponding to the saddle point with higher order instability index has also been numerically checked to be satisfied for each superlinear elliptic equation computed in [20, 21].

Next we consider the case where

$$
\langle A u, u\rangle=\int_{\Omega}\left(|\nabla u(x)|^{2}-\lambda u^{2}(x)\right) d x
$$

where $\lambda_{k}<\lambda<\lambda_{k+1}$ and $\lambda_{k}$ is the $k$-th eigenvalue of the Laplacian operator $-\Delta$. Thus we have $H^{0}=\{0\}$ and $H^{-}=\left\{u_{1}, \ldots, u_{k}\right\}$ where $u_{1}, \ldots, u_{k}$ are the eigenfunctions of $-\Delta$ corresponding to the first $k$ eigenvalues $\lambda_{1}, \ldots, \lambda_{k}$. When $f$ is superlinear, it is clear that $\operatorname{MI}(0)=k$. Thus we set $L^{0}=\left\{u_{1}, \ldots, u_{k}\right\}$. First let $L=\{0\}$ and let the support $L^{1}=L^{0} \oplus L$. Then we apply the local minimax method to find a saddle point $u^{*}$. The instability index of $u^{*}$ relative to 0 is $\operatorname{dim}(L)+1=1$. Next we set $w_{1}=u^{*}$ and $L=\left\{w_{1}\right\}$, use the local minimax method with the support $L^{1}=L^{0} \oplus L$ to find a new saddle point whose instability index relative to 0 will be $\operatorname{dim}(L)+1=2$, etc. [33]

As a last example, we consider a semilinear Schrodinger equation of the form

$$
-\Delta u+V(x) u=f(x, u), \quad u \in H^{1}\left(\mathbb{R}^{n}\right),
$$

where $V$ and $f$ are periodic with respect to $x, 0$ lies in a gap of the spectrum of the operator $A: u \rightarrow-\Delta u+V(x) u$ and $f(x, 0)=0$ satisfies some standard regularity and growth conditions [33] to guarantee the existence of multiple nontrivial solutions. The corresponding generic energy functional is

$$
J(u)=\langle A u, u\rangle-\int_{\mathbb{R}^{n}} F(x, u) d x
$$

where

$$
\langle A u, u\rangle=\int_{\mathbb{R}^{n}}\left\{\frac{1}{2}|\nabla u|^{2}+\frac{1}{2} V(x) u^{2}\right\} d x \quad \text { and } \quad F(x, t)=\int_{0}^{t} f(x, \tau) d \tau .
$$

Then $H \equiv H^{1}\left(\mathbb{R}^{n}\right)=H^{-} \oplus H^{+}$, where $H^{-}$and $H^{+}$are, respectively, the infinitedimensional maximum negative and positive definite subspaces of the operator $A$. It is clear that 0 is a trivial solution with $\mathrm{MI}(0)=+\infty$. As a matter of fact, any solution has $\mathrm{MI}=+\infty$. Thus we cannot distinguish their local instabilities by using their Morse indices or (weak) local linking indices. However, by our formulation above, we can set $L^{0}=H^{-}$and use our local minimax method with a support $L^{1}=L^{0} \oplus L$ for some closed subspace $L \subset H^{+}$. If we can find a local minimax solution $u^{*}$, then the local minimax index of $u^{*}$ relative to the trivial solution 0 is $\operatorname{dim}(L)+1$. Thus local instability analysis of different solutions can be carried out.

\section{REFERENCES}

1. A. Ambrosetti and P. Rabinowitz, Dual variational methods in critical point theory and applications, J. Funct. Anal. 14 (1973), 349-381. MR 51:6412

2. A. Bahri and P.L. Lions, Morse index of some min-max critical points. I. Application to multiplicity results, Comm. Pure Appl. Math., Vol. XLI (1988) 1027-1037. MR 90b:58035

3. A. Bahri and P.L. Lions, Solutions of superlinear elliptic equations and their Morse indices, Communications on Pure and Applied Math., 45 (1992), 1205-1215. MR 93m:35077 
4. T. Bartsch and Z.Q. Wang, On the existence of sign-changing solutions for semilinear Dirichlet problems, Topol. Methods Nonlinear Anal. 7 (1996), 115-131. MR 97m:35076

5. T. Bartsch, K. C. Chang and Z. Q. Wang, On the Morse indices of sign changing solutions of nonlinear elliptic problems, Math. Z., 233 (2000), 655-677. MR 2001c:35079

6. H. Brezis and L. Nirenberg, Remarks on Finding Critical Points, Communications on Pure and Applied Mathematics, vol. XLIV, (1991), 939-963. MR 92i:58032

7. K.C. Chang, Infinite Dimensional Morse Theory and Multiple Solution Problems, Birkhäuser, Boston, 1993. MR 94e:58023

8. Y. S. Choi and P. J. McKenna, A mountain pass method for the numerical solution of semilinear elliptic problems, Nonlinear Analysis, Theory, Methods and Applications, 20 (1993), 417-437. MR 94c:65133

9. Y. Chen and P. J. McKenna, Traveling waves in a nonlinearly suspended beam: Theoretical results and numerical observations, J. Diff. Equ., 136 (1997), 325-355. MR 98g:73014

10. C.V. Coffman, A nonlinear boundary value problem with many positive solutions, J. Diff. Eq., 54 (1984), 429-437. MR 86e:35055

11. E.N. Dancer, The effect of domain shape on the number of positive solutions of certain nonlinear equations, J. Diff. Eq., 74 (1988), 120-156. MR 89h:35256

12. W.Y. Ding and W.M. Ni, On the existence of positive entire solutions of a semilinear elliptic equation, Arch. Rational Mech. Anal., 91 (1986), 238-308. MR 87b:35056

13. Z. Ding, D. Costa and G. Chen, A high linking method for sign changing solutions for semilinear elliptic equations, Nonlinear Analysis, 38 (1999) 151-172. MR 2000d:65208

14. J.J. Garcia-Ripoll, V.M. Perez-Garcia, E.A. Ostrovskaya and Y. S. Kivshar, Dipole-mode vector solitons, Phy. Rev. Lett., 85 (2000), 82-85.

15. J.J. Garcia-Ripoll and V.M. Perez-Garcia, Optimizing Schrodinger functionals using Sobolev gradients: Applications to quantum mechanics and nonlinear optics, SIAM Sci. Comp., 23 (2001), 1316-1334. MR 2002k:35266

16. I. Kuzin and S. I. Pohozaev, Entire Solutions of Semilinear Elliptic Equations, Birkhauser, Boston, 1997.

17. A. C. Lazer and S. Solimini, Nontrivial solutions of operator equations and Morse indices of critical points of min-max type, Nonlinear Analysis, 12 (1988), 761-775. MR 89i:58018

18. J. Q. Liu and S. J. Li, Some existence theorems on multiple critical points and their applications, Kexue Tongbao, 17 (1984).

19. S.J. Li and M. Willem, Applications of local linking to critical point theory, J. Math. Anal. Appl., 189 (1995), 6-32. MR 96a:58045

20. Y. Li and J. Zhou, A minimax method for finding multiple critical points and its applications to semilinear PDE, SIAM J. Sci. Comp., 23 (2001), 840-865. MR 2002h:49012

21. Y. Li and J. Zhou, Convergence results of a local minimax method for finding multiple critical points, SIAM J. Sci. Comp., 24 (2002), 865-885. MR 2004a:58013

22. Y. Li and J. Zhou, Local characterization of saddle points and their Morse indices, Control of Nonlinear Distributed Parameter Systems, pp. 233-252, Marcel Dekker, New York, 2001. MR 2002c: 49048

23. Y.Y. Li, Existence of many positive solutions of semilinear elliptic equations on annulus, J. Diff. Eq., 83 (1990), 348-367. MR 91a:35073

24. J. Mawhin and M. Willem, Critical Point Theory and Hamiltonian Systems, Springer-Verlag, New York, 1989. MR 90e:58016

25. Z.H. Musslimani, M. Segev, D.N. Christodoulides and M. Soljacic, Composite Multihump vector solitons carrying topological charge, Phy. Rev. Lett., 84 (2000) 1164-1167.

26. Z. Nehari, On a class of nonlinear second-order differential equations, Trans. Amer. Math. Soc., 95 (1960), 101-123. MR 22:2756

27. W.M. Ni, Some Aspects of Semilinear Elliptic Equations, Dept. of Math., National Tsing Hua Univ., Hsinchu, Taiwan, Rep. of China, 1987.

28. W.M. Ni, Recent progress in semilinear elliptic equations, in RIMS Kokyuroku 679, Kyoto University, Kyoto, Japan, 1989, 1-39.

29. P. Rabinowitz, Minimax Method in Critical Point Theory with Applications to Differential Equations, CBMS Regional Conf. Series in Math., No. 65, AMS, Providence, 1986. MR 87j:58024

30. E. A. Silva, Multiple critical point for asymptotically quadratic functionals, Comm. Part. Diff. Equ., 21 (1996), 1729-1770. MR 97m:58037 
31. J. Smoller, Shock Waves and Reaction-Diffusion Equations, Springer-Verlag, New York, 1983. MR 84d:35002

32. S. Solimini, Morse index estimates in min-max theorems, Manuscripta Math., 63 (1989), 421-453. MR 90f:58028

33. C. Troestler and M. Willem, Nontrivial solution of a semilinear Schrodinger equation, Commun. Part. Diff. Equat., 21 (1996), 1431-1449. MR 98i:35034

34. Z. Wang, On a superlinear elliptic equation, Ann. Inst. Henri Poincare, 8 (1991), 43-57. MR 92a:35064

35. M. Willem, Minimax Theorems, Birkhauser, Boston, 1996. MR 97h:58037

36. J. Zhou, A min-orthogonal method for finding multiple saddle points., J. Math. Anal. Appl., 291 (2004), 66-81.

Department of Mathematics, Texas A\&M University, College Station, Texas 77843

E-mail address: jzhou@math.tamu.edu 